\title{
The analysis of vibration response of air-cooled refrigerator excited by reciprocating compressor
}

\author{
Huaiqian $\mathrm{Bao}^{1,2}{ }^{*}$, Baokun Han ${ }^{1}$, Yufeng $\mathrm{Xu}^{2}$, Yuechao Liu ${ }^{2}$, Guifang Liu ${ }^{1}$ \\ ${ }^{1}$ Shandong University of Science and Technology, Mechanical and Electronic Engineering, Shandong Qingdao \\ ${ }^{2}$ Qingdao AUCMA Corporation, Shandong Qingdao
}

\begin{abstract}
The features of large-capacity and multi temperature zones of air-cooled refrigerators are favored by consumers. However, the vibration of different components caused by the vibrations of different components in the operation process brings certain troubles to people's lives. In order to achieve better vibration and noise reduction of the refrigerator, the vibration characteristics of the air-cooled refrigerator are analyzed under the excitation of compressor, selects four detection points, and uses simulation methods to obtain the excitation of the compressor. In addition to the impact of the vibration source on the refrigerator, the assembly of refrigerator door is also an important factor of the refrigerator vibration. The experimental process has also found that the air-cooled system also has an impact on the vibration and noise generation of the refrigerator.
\end{abstract}

\section{Introduction}

With the rapid development of household appliances, most brands have gradually emerged from the low-cost expansion model and have turned to the high-end product market. For domestic refrigerators, there are two main forms, direct-cooled and air-cooled frost-free refrigerators. Direct-cooled refrigerators have not reached the increasing demand for high-capacity and multi temperature zones for refrigerators. The air-cooled refrigerator adopts fan-assisted cooling, which can realize people's various demands on the refrigerator. However, although the air-cooled refrigerator saves the cost and meets the demand for cooling capacity, the multiple machines run under the working state, resulting in vibration and noise are far greater than air-cooled refrigerators, which brings convenience to people and also brings certain problems.

\section{Analysis of vibration characteristics of reciprocating compressor}

During the working process of air-cooled refrigerators, the sources of vibrations are various. For example, the vibration of compressor, the vibration of fan, and the secondary vibration excitation of the pipeline can be transmitted to refrigerator, causing vibration of the refrigerator. This article selects compressor as vibration source and analyzes vibration response of the air-cooled refrigerator.

The refrigerator compressors are most commonly used in the market that mostly reciprocating. The reciprocating compressors have advantages of low thermal efficiency, convenient processing, low material requirements, low manufacturing cost, and wide application range. It is mainly composed of cylinder, piston and gas valve. The gas completes the work of compressor through four processes of expansion, suction, compression and exhaust. Complex internal moving parts cause many sources of vibration that cause vibration and noise in the compressor. Among them, the incentives that cause vibration noise of compressor are divided into four categories: inertial force, gas impact force, mechanical impact and frictional force. According to a large number of documents, inertial forces play a major role in the generation of compressor vibrations. Therefore, when analyzing the vibration characteristics of a compressor, only the effect of inertial force is considered. Figure. 1 shows the structure of the crank connecting rod that causes the vibration of reciprocating compressor.

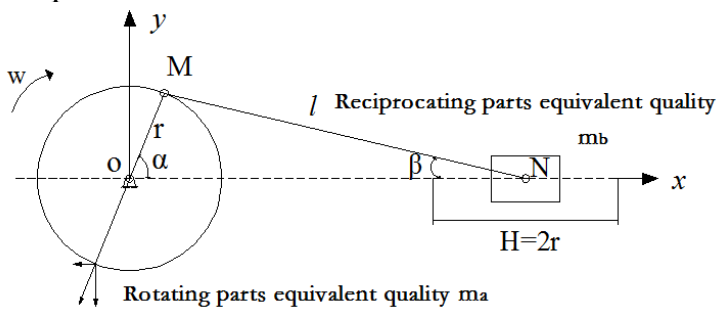

Fig.1. Reciprocating compressor internal crank linkage

The reciprocating inertial force is generated by the reciprocating member, it generates the reciprocating inertial force:

$$
F_{b}=m_{b} \ddot{x}=m_{b} l \omega^{2} \cos \alpha+m_{b} l \omega^{2} \lambda \cos 2 \alpha
$$

In the formula, $m_{b}$ is the equivalent mass of the reciprocating part (piston, connecting rod small head, cross head); $\alpha$ is the rotation angle of the crank, $\alpha=(\omega t+\varphi) ; \varphi$ is the initial phase angle; $\lambda=r / l$. 
The rotational inertia force is caused by the unbalance of the mass of the moving crankshaft. The rotational inertia force generated by the entire system.

$$
F_{a}=m_{a} r \omega^{2}
$$

In the formula, $m_{a}$ is the equivalent total mass of rotary motion resulting from conversion of crankshafts, connecting rods, etc. $r$ is the radius of rotation of the crank; $\omega$ is the angular speed of crank rotation.

The total force generated by the inertial force on the reciprocating compressor

$$
F_{1}=F_{a}+F_{b}
$$

Simplified vibration models for reciprocating compressors and air-cooled refrigerators are shown in Figure2.

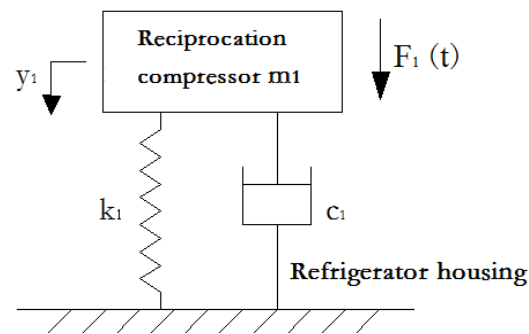

Fig. 2. Reciprocating Compressor - Single-degree-of-freedom vibration model of air-cooled refrigerator

The equivalent mass of the compressor core and housing is defined as $m_{1}$, and the four rubber pads of the four compressor feet that are in contact with the bottom support plate of the refrigerator, and define the equivalent stiffness $k_{1}$ and equivalent damping $c_{1}$. differential equation of motion for reciprocating compressor-air-cooled refrigerator housing was established.

$$
m_{1} \ddot{y_{1}}+c_{1} \dot{y_{1}}+k_{1} y_{1}=F_{1}
$$

In the formula, $F_{1}$ is the force generated by the internal structure of the compressor on the compressor.

\section{Analysis of vibration response of air-cooled refrigerator}

Dynamic response analysis of structures is a technique for determining the steady-state response of a linear structure subjected to a load that changes sinusoid ally over time. Harmonic response analysis enables technicians to better predict the continuous dynamic characteristics of the structure and ensure that the technical designer can better predict whether the product can overcome the harmful effects of resonance and improve the quality performance of the product. In the harmonic response analysis process, the four positions of the air-cooled refrigerator are selected as the detection points of the vibration response. The positions of the selected measuring points are: the measuring point is the junction between the refrigerator door and the drawer; the measuring point is the left side of the refrigerator The surface position; the measuring point three is the installation fan position; the measuring point four is the supporting plate on the bottom of the refrigerator. The simulation results are shown in Figure. 3.

According to the method of Newton's second law, a

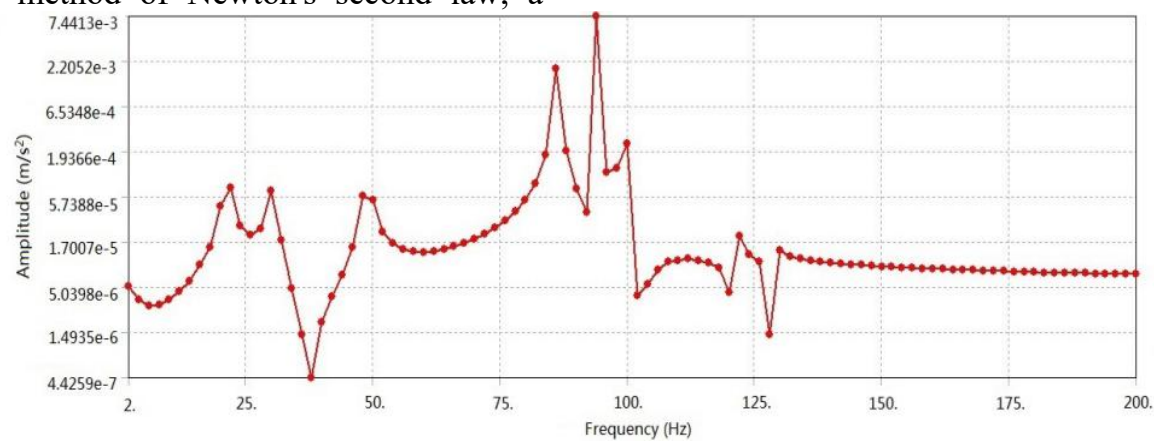

(a) Fridge door and drawer joints

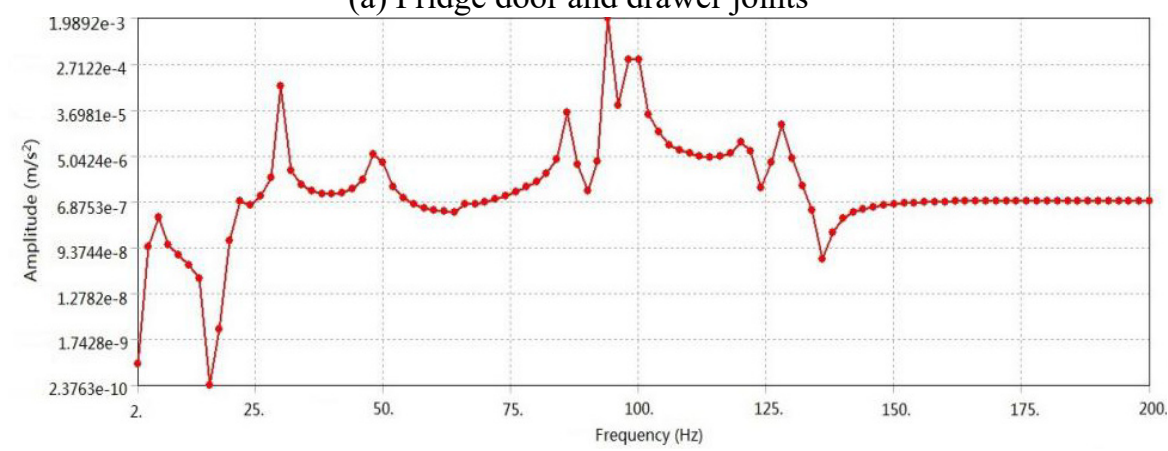

(b) The left side surface of the refrigerator 


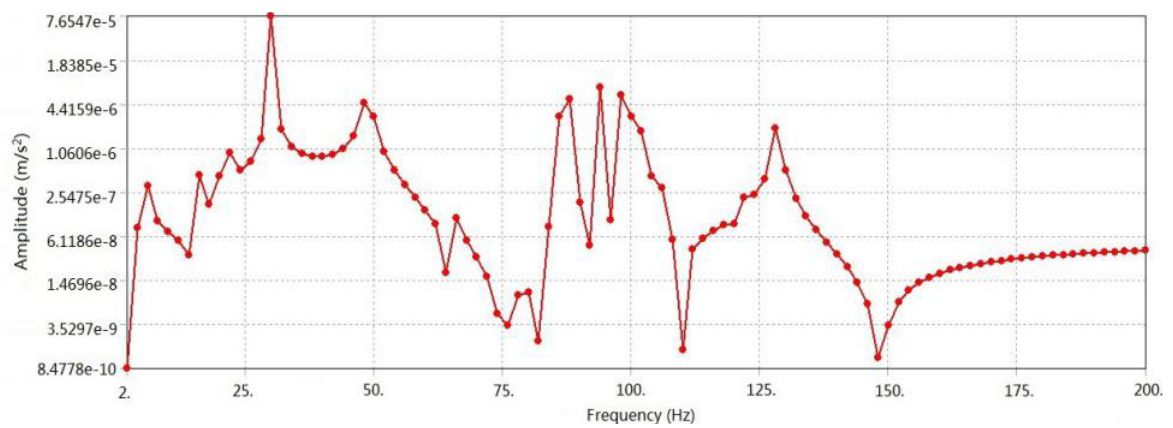

(c) Install fan position

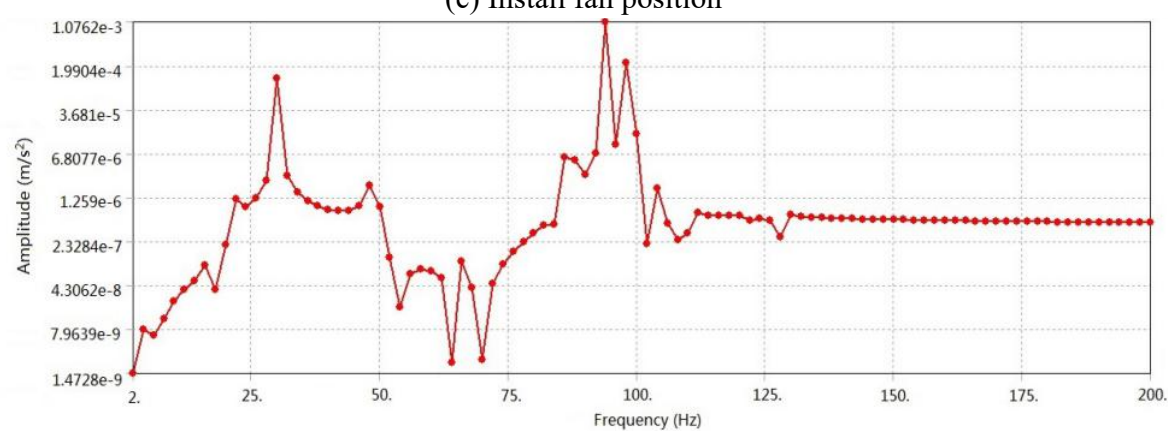

(d) Compressor around refrigerator bottom support plate

Fig. 3. Compressor vibration source excitation, air-cooled refrigerator vibration response simulation results

The maximum vibration response amplitudes at the when the compressor is adjusted are shown in Table 1. four measurement points of the air-cooled refrigerator

Table1. Maximum vibration response amplitude at four measurement points of an air-cooled refrigerator

\begin{tabular}{ccccc}
\hline Check point & Measure one & Measure two & Measure three & Measure four \\
\hline Frequency $(\mathrm{Hz})$ & 93 & 93 & 30 & 93 \\
Amplitude $\left(\mathrm{m} / \mathrm{s}^{2}\right)$ & $7.44 \times 10^{-3}$ & $1.99 \times 10^{-3}$ & $7.65 \times 10^{-5}$ & $1.08 \times 10^{-3}$ \\
\hline
\end{tabular}

\section{Vibration-response experiment of air-cooled refrigerator}

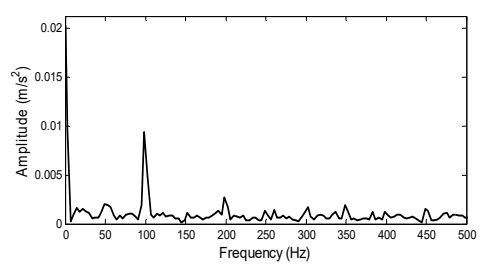

(a) Measuring point one

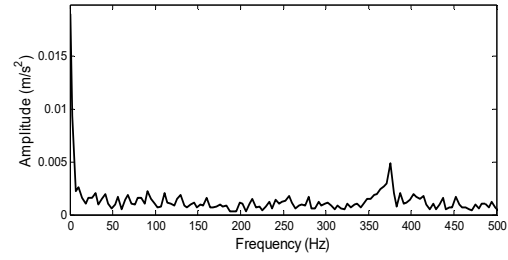

(b) Measuring point two
The experimental study on the vibration response of the air-cooled refrigerator when the compressor was operated alone was performed using the LMS vibration measurement system. The experimental results of the vibration response were shown in Figure4.

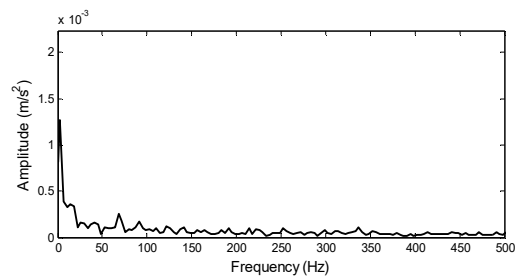

(c) Measuring point three

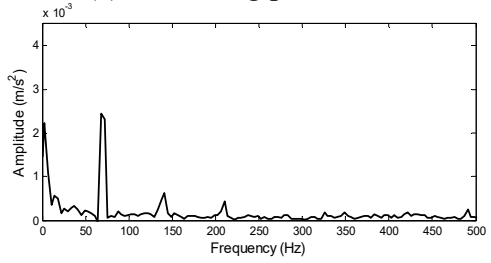

(d) Measuring point four

Fig. 4 Vibration response test results of an air-cooled refrigerator case when the compressor is operated separately 
The maximum vibration response amplitudes at the four measuring points are shown in Table2.

Table 2. Maximum vibration response test results at four measurement points when the compressor is operated separately

\begin{tabular}{ccccc}
\hline Check point & Measure one & Measure two & Measure three & Measure four \\
\hline Frequency $(\mathrm{Hz})$ & 100 & 90 & 70 & 70 \\
Amplitude $\left(\mathrm{m} / \mathrm{s}^{2}\right)$ & $9 \times 10^{-3}$ & $1.75 \times 10^{-3}$ & $2.5 \times 10^{-3}$ & $7 \times 10^{-4}$ \\
\hline
\end{tabular}

The maximum vibration response amplitude at 100 $\mathrm{Hz}$ at the measuring point is $9 \times 10^{-3} \mathrm{~m} / \mathrm{s}^{2}$, The maximum vibration response amplitude at point $90 \mathrm{~Hz}$ at the measuring point two is $1.75 \times 10^{-3} \mathrm{~m} / \mathrm{s}^{2}$, The maximum vibration response amplitudes at $70 \mathrm{~Hz}$ in the three and four measuring points are $2.5 \times 10^{-3} \mathrm{~m} / \mathrm{s}^{2} ; 7 \times 10^{-4} \mathrm{~m} / \mathrm{s}^{2}$.

\section{Conclusion}

Compared with the experimental simulation results, it can be found that the frequency of the maximum vibration response amplitude at the measurement point is similar and the vibration response amplitudes of both are larger than the other three measurement points, Therefore, in the research of air-cooled refrigerator damping and noise reduction, the processing and assembly of the refrigerator door is a non-negligible factor. The second point of vibration at the measuring point originates from the vibration transmission of the compressor; the difference between the three measuring points is relatively large, mainly because the measuring point three is the location of the installed fan. Although the fan stops running during the experiment, the actual process still has an effect, causing the frequency of the maximum vibration response amplitude to shift back, and the experimental results are obviously high.

\section{References}

1. Yang. X. X. Technology Information, 12( 2014) 83-83.

2. Ma. C. Z. Daily appliances, 10(2017) 43-46.

3. Dong. C. Q.Research and safety evaluation of the mechanical performance of the whole reciprocating compressor crankshaft. Southwest Petroleum University (2014)

4. Chu. C. G. Parts Manufacturing Technology, (2012) 463-464

5. Yang. B. Dynamic response analysis of 8L625 air frame under multiple excitation. Dalian University of Technology, (2015). 